\title{
Effects of learners' characteristics and instructional guidance on computer assisted learning
}

\author{
Qi Chen
}

Department of Psychology

Beijing Normal University, China

\begin{abstract}
Research has shown that only under certain conditions can CAI or other media function more effectively. This paper summarizes, on the basis of our several studies in secondary schools, how the variables such as instructional guidance, learner's learning style, and the extent of learner control moderate the effects of computer use in classrooms. Learners are the masters in the learning process and the inner factor of their mental development. Only through the learners' mediation can the technical potential be realized. Only those teachers who have mastered an understanding of the students' learning processes are aware of the conditions under which the new technology can play its role efficiently.
\end{abstract}

Keywords: teaching methods, courseware, learning models, learning skill, motivation. 


\section{INTRODUCTION}

\section{The Chinese education system}

In the Chinese system, the central government outlines educational principle and policies through the State Education Commission which is the highest educational administrative institution and is controlled directly by the State Council. After many years of exploration, and especially the recent decade's experience, the Chinese education system has been undergoing a process of continuous reform and readjustment. A whole new set of systems has come into being. General education is mainly publicly funded; however, privately-funded schools have been permitted.

Although the pre-school education has been developed rapidly in China, it is not compulsory. The nine-year compulsory education that includes primary and junior secondary has been implemented step by step in a planned way, starting from 1985 [1]. The schooling system, including primary, junior and senior secondary, is mainly a 6-3-3-year system. In some regions, 5-4-3 or 5-3-3-system is implemented instead. The policy or principle for education is to enable students to get all-round development in moral, intellectual, physical and aesthetical aspects. The graduates of junior high schools must take an entrance examination held by local educational bureaus to enter senior secondary education, including professional and polytechnic schools. The graduates of senior secondary have to take the unified national admissions examination for high education. Because of a limited quota for studying, only a small percentage of senior high graduates are admitted to the universities and colleges.

Curriculum reform has been progressing rapidly. It used to be unified for the whole country either in syllabus or in textbook. Now it has been changed to "one syllabus but multitexts". One syllabus means the basic requirement for each subject should be met to ensure the quality of schooling, while multitexts meet different needs of different areas. Since China is such a big country and the developments in different areas have been so unbalanced, this reform policy gives local educational bureaus more flexibility to design their own texts to meet the local needs. The curriculum structure in senior secondary education also has been reformed to be more flexible. More free elective courses which are more practical and related to vocational education have been offered instead of some required courses. In addition, some activity courses have been offered to meet the needs of situated learning. 


\section{Description of educational computing in the classroom}

In China, educational computing in secondary schools dates back to 1982 . Learning about the computer, mainly learning programming languages, has been the core of computer education in secondary schools [2]. Since 1986, the State Education Commission along with other related institutions, has realized that to integrate computer into curricula should be the trend of educational computing [3]. While financial funds were being allocated for software development, special teams were organized to develop educational software. At the same time an educational software evaluation committee was established. As a result, computer assisted instruction was greatly encouraged. During its rapid development, educators also realized that as the core of modern technology, the use of computer in instruction would be an inevitable trend.

Nevertheless, there have been several different perspectives on the functions of CAI in schools. Schools which had advanced educational perceptions and ideas about the educational reform encouraged their best teachers to use the computer as an instructional medium to improve their teaching quality. The best teachers, programmers, and curriculum designers were brought together to develop courseware. Pilot studies and experiments were conducted, and, after those pilot studies, experimentation had been conducted, and, after these pilot studies, further experimentation took place and this led to an expansion of the implementation. Unfortunately, only a few schools held this type of perspective on CAI.

Some schools or regions are very enthusiastic towards CAI and adopted it completely. But they regarded information technology as a "fashion", they had no theoretical concepts about how educational technology should function well and what has happened in the world of information technology. The effects of CAI have often been so exaggerated as a kind of panacea for educational reform that CAI itself has become not an approach but an objective in teaching. It seems they believed that as long as CAI was used, its potential power would be automatically harnessed. Enthusiasm towards computer use in education should be protected and encouraged, but it is possible that some theoretical training about CAI should be given to those educators who hold this unquestioning enthusiasm.

A third perspective is found in those schools that are well-equipped with computers but without any knowledge or enthusiasm towards educational computing at all. Sometimes they just exhibit their computers to the visitors, or only teach about the computer. 


\section{Integrating information technology into education}

\section{RESEARCH FINDINGS}

The impact of integrating information technology into education has been investigated in the past three decades. The initial positive fever for computer education has now been replaced by a more tempered attitude. It appears that CAI is relatively more effective only under certain conditions. Variables pertaining to learners, instruction, and software should be examined systematically [4]. This paper summarizes from our several studies, how those variables, such as learning style, the extent of learners' control and instructional guidance, could moderate the effects of educational computing.

\section{Teacher's guidance and learner's characteristics}

To examine how the extent of teacher's guidance moderates the effects of CAI, a series of studies on Computer Assisted English Learning were conducted in three secondary schools [5]; GuangDong Experimental Middle School in GuangZhou, GuangDong, P.R.C., (hereafter referred to as School A), ZhuHai \# 1 Middle School in ZhuHai, GuangDong, P.R.C. (referred to as School B), and Workers' Children School in Macau (referred to as school C). Two Form Four classes, one for experimental group and one for control, were randomly selected in each of these schools as the study target. The experimental group used the computer assisted English learning, while the control group was taught the same content by an English teacher. The same educational software was used in each of these schools' experimental groups, but with different degrees of instructional guidance. In School A, the teacher gave the meaning of most of the vocabularies to the students (Most Guidance), while in school C, the teacher was told to let the students themselves explore the meaning of the vocabularies (Least Guidance). In School B, the instructor provided the students with the meaning of the key words only (Moderate Guidance). The first stage results showed that the experimental group in School C exhibited significant differences from their control counterparts in their final achievement. There were no significant differences in their achievement between the experimental and control classes in the other two schools.

Examination of the experimental procedure indicated that, despite the initial effort to standardize the conditions and procedure of the study in these three schools, students in School A appeared to be more motivated and of higher standard to start with, and that the experimenter (i.e., teacher) in School B was less enthusiastic than his counterparts in the other two schools. Consequently two supplementary studies were 
conducted. In School A, software of a more difficult level than the original one was used. In School B, the experiment was conducted by an experimenter whose instructional behaviour was trained to be comparable to that of the other two schools. The final achievement showed some improvement of those two experimental groups, though the differences were still not significant. Our preliminary conclusion for this aspect of teachers is that less guidance could induce greater student achievement [5].

Contrary to what was found for achievement, a measure of attitude indicated, that the target students in School A and B harboured a much more positive attitude and expectation toward Computer Assisted Learning than those in School $\mathrm{C}$, though their achievements lagged far behind. They believed that the more they learned, the more their capacity for self-learning would be fostered, and the more they were willing to undergo Computer Assisted Learning in future. We found that the characteristics of students, including cultural background and level of motivation, are as relevant when considering the use of CAI as the extent of teacher guidance.

The students in School A and B were strictly selected by a unified entrance examination. Both students in experimental group and control group were well-motivated and had quite a high level in English study. Because of the high starting point of those students, it might be hard to show the differences in English achievement using different means in merely six weeks. More needs to be known about the processes leading to our research result that more guidance induced less visible achievement but a more positive attitude. Perhaps with more guidance, students felt the task was more attainable, resulting in a more assured confidence in learning with computer. It is also plausible that with more guidance, students could focus on other aspects of learning with the computer, thus raising their interest in such medium of learning.

This study focussed on one aspect of instruction. The extent that other aspects of instruction, such as the time given to students to interact with the computer, which might affect students' learning, attitude, and perception in different ways also should be examined. Equally important are variables related to the learners, which may interact with style of instruction to affect their learning and attitude.

\section{The extent of learner control and cognitive style}

To examine the effects of types of instructional control and cognitive style on tutorial CAI, a three by three matrix design was used; program adaptive control/ learner control/ learner with advice control were considered 
against field dependence/ middle level/ field independence [6]. Results indicated that learner control and learner with advice control were more effective than program adaptive control. Although there were no significant differences between these two effective groups, learner with advice control was slightly better than learner control.

Learning with the computer, as a relatively newer medium of instruction, appears to call for more participation on the part of the learners. In effect, an instructional style that grants more freedom and opportunity to the learner might induce better learning and a more positive attitude than a more controlled style of instruction. In these two learner control groups, students could not only control the content of learning, that is, the sequences of learning materials and examples, but also control the learning strategies such as the way of presentation and the quantity of practice. They could use the freedom which was provided through learner control and had to make their own learning decisions, therefore, spending more time learning than the program adaptive control group. In addition, while these students were progressing at their own pace, there was no doubt that these students could master all the content they had to learn. The results also showed that the variable of the extent of learner control has significantly interacted with learner's learning style. Field independent students performed better in the way of learner control, while field dependent students were better with program adaptive control and the middle students performed the best in the role of learner with advice control.

This study has explored CAI with a content of a moderate level of difficulty, mainly including conceptual learning. More research is needed to explore which extent of learner control under different levels of difficulties would be more effective.

\section{Learning skills training and cognitive styles}

Further exploration about the effects of different types of guidance on learning skills, cognitive styles, and the model of learner control have been conducted [7]. The task of this study was for students to learn how to use Chinese punctuation through CAI. Three groups of 143 students in total had been randomly selected from first year of junior high school. Each group was treated differently. Group A was learning Chinese punctuation following the procedure which was prescribed by the courseware itself. No tutorial of learning skills was involved in. Group B was learning through the courseware which had some tutorials built-in, that is, hints or cues for key words and learning skills. Group C, however, had teacher's guidance in addition to the courseware with 
tutorial. This meant that the teacher taught one or two examples in the beginning and pointed out the focus, explained the difficult points and reminded students of the important feedback from the computer. Also, during the learning period, the teacher walked around the classroom, answered questions raised by the students, and provided some advice of learning methods.

Results showed that Group C, using courseware with tutorials combined with teacher's guidance, gained the best achievement, obtained the best effect on transfer, and used less time than others. Considerable interaction between the cognitive style of students and the transfer effect on CAI of the Chinese punctuation was indicated. The transfer effect of field independent students proved better than that of field dependent students, especially in their achievement of the post-test. The results were quite clear. After they had really grasped the propositions and rules about the Chinese punctuation, they were able to use them better; even though they were given a substantial problem in their learning situation, their success was quite consistent as well as the transfer effect. This demonstrated that they could take advantage of their inner reference to make their judgment independently. Group $\mathrm{C}$ and Group B were both involved with guidance on learning skills, with the implied elements of meta-cognition; hence their effect of transfer should be more conspicuous.

\section{CONCLUSIONS}

From these experimental research studies, we have not yet made an all-round and detailed analysis on the variables and parameters in the experimental program. We have so far only selected the findings on learning characteristics of students themselves and the teachers' guidance, in order to offer some facts. We want to show that these two variables are of great importance in their influences on the effectiveness of CAI. The special features of students include their cultural background, learning basis, learning style, motivation, and their expectation in the learning environment offered by CAI. All of these can have an influence on the learning effect. Students themselves are the masters in the learning process, also the inner factor of their development. No matter how advanced a technique, a method, or a measure is, only through the student who is the vital factor as the mediator in the learning environment, can such a resource realize its potential function. In this process, there's no exception, even with information technology and the computer as its core. 
This point is what our experimental researches have indicated time and again.

Starting from John Dewey, some western educational theorists have emphasized the child-centred education in regard to the statement above. I agree with this stand-point. But I do not entirely agree with the child-centrists because the primary and secondary students are growing individuals. Whether in terms of their socialization or of their development in knowledge, intelligence, and technical ability, they have to experience the process from immaturity to maturity, from unknowing to knowing. "Learning by doing" is a quite good method as they learn from their direct experience, for it can meet the individual needs to learn at his/her own pace. However, it is neither possible nor necessary for them to learn by experiencing everything. For one of the special features of student learning is to learn mainly from indirect experiences, and here, the teacher's guidance still plays a very important role. The teacher's guidance can protect students from wasting time on "trial and error" in their learning process, help them to speed up their process of information processing and thus gradually raise the quality of this process. With all the above, our three studies have proved this point. Though it is not immediately clear in the first experiment that the teacher's guidance has a positive effect on the students' achievement, it can be seen definitely from the inquiry on attitude. In the other two studies, learner control with guidance and courseware guidance combined with teacher's guidance both had a positive effect.

Thus the "outer condition", especially the teacher, cannot be neglected. The inner factor is the base of learning, the outer factor the condition of learning, the outer factor functions through the inner. This is a famous saying by Mao Ze-dong [8]. It is ultimately appropriate to cite here to explain the relationship between child-development and education, the relationship between teaching and learning. It is natural that with the growing and maturing of psychological development, the teacher's guidance can be gradually simplified and reduced. But the appropriate amount and the extent of this guidance still needs to be explored. Convincingly, even adults need necessary guidance in the process of learning new knowledge and thus complete the leap from unknowing to knowing. According to Piaget's theory of cognitive development, some adults might not reach the formal operation stage even in the life long time. Therefore, the training on learning skill is not only beneficial to the learning process of students, but also advantageous to the adults' learning.

I believe that the integration of information technology into the instructional process develops an immense potential for the student's 
learning environment. However, the technology itself cannot automatically release its potential; only teachers who have mastered the student's learning patterns are aware of the conditions under which this new technology can play its role efficiently. Then a student's learning and development can be promoted consciously.

\section{REFERENCES}

1. (1990) Encyclopedia of Chinese Education, Chinese Encyclopedia Press.

2. Chen, Q. (1990) Seven Years' Retrospect and Prospect: Computers in Chinese Secondary Education. McDougall, A. and Dowling, C. (eds) Computers in Education: Proceedings of the 5th World Conference on Computers in Education. IFIP, Elsevier Science Pub. Co., North-Holland.

3. Chen, Q. editor (1987) Readings On Computer Education in Secondary Schools. GuangMing Daily Press.

4. Chen, Q. (1991) Variables Moderating the Effect of Computer in Education. Proceedings of IFIP WG3.1 Working Conference, UCSB. Elsevier Science Pub. Co., North-Holland

5. Chen, Q. (1992) Effects of Computer Assisted English Learning on Students' Achievement and Attitude. Proceedings of The Second Afro-Asia Psychological Congress, Beijing University Press.

6. Xia, W.F. \& Chen, Q. (1991) The Effects of Types of Instructional Control and Cognitive Styles on Tutorial CAI. (in press)

7. Qing Z. J. \& Chen, Q.(1992) An Experimental Study on Learning Chinese punctuation through Computer.Assisted Instruction. (in press)

8. Mao, Z.D. (1943) On Practice. Selected Works of Mao ZeDong. People's Publishing House. 


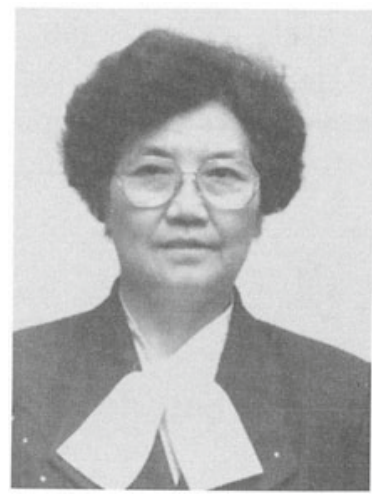

Qi Chen, formerly Dean of Faculty of Education at the University of Macau, is now Professor of Educational Psychology in the Department of Psychology, Beijing Normal University and Deputy Director of the National Research Centre on Computer Education in Schools. She is also a member of the Board of Directors of the Chinese Psychological Society and vice president of the Educational Psychology Committee. Qi Chen was a visiting scholar in Psychology and Education at UCLA during 1980-83 and has been doing research on educational computing for 10 years. She believes that with more understanding about student learning, information technology could be more effectively integrated into education. 